Total joint replacements

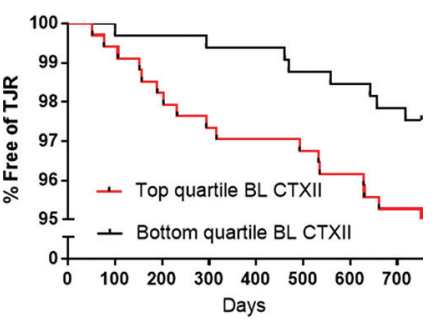

Abstract THU0419 - Figure 1. TJR events during the entire study, CTX-II.

Conclusion: High levels of SCTX-I and UCTX-II at BL were associated with increased risk of undergoing TJR of the knee or hip during the two important biomarkers in clinical OA trials evaluating incidence of TJRs.

Disclosure of Interests: : Jonathan Bjerre-Bastos Employee of: I am a full-time employee in Nordic Bioscience, Anne-Christine Bay-Jensen Shareholder of: I own shares of Nordic Bioscience, Employee of: I am a fulltime employee in Nordic Bioscience, Morten Karsdal Shareholder of: I own shares of Nordic Bioscience, Employee of: I am a full-time employee in Nordic Bioscience, Inger Byrjalsen Employee of: I am a full-time employee in Nordic Bioscience, Jeppe Ragnar Andersen Shareholder of: Nordic Bioscience, Employee of: Nordic Bioscience, Bente Juel Riis Shareholder of: Yes, I own shares in Nordic Bioscience, Employee of: I am a full-time employee in Nordic Bioscience, Claus Christiansen Shareholder of: Yes, I own shares in Nordic Bioscience, Employee of: I am a full-time employee in Nordic Bioscience, Asger Reinstrup Bihlet Shareholder of: Yes, I own shares in Nordic Bioscience., Employee of: I am a full-time employee in Nordic Bioscience

DOI: 10.1136/annrheumdis-2019-eular.7306

\section{THU0420 METABOLIC SYNDROME AND TRAJECTORIES OF LOCALISED PAIN AND GENERALISED PAIN}

Feng Pan ${ }^{1}$, Jing Tian ${ }^{1}$, Flavia Cicuttini ${ }^{2}$, Graeme Jones ${ }^{1} .{ }^{1}$ University of Tasmania, Medical School, Department of Epidemiology and Preventive Medicine, Melbourne, Australia

Background: Metabolic syndrome (MetS) has been suggested as having a link to the pathophysiology of pain; however, no study has assessed generalised pain and their courses over time.

Objectives: To describe the associations of MetS and its components with trajectories of localised knee pain (pain severity) and generalised pain ((number of painful sites (NPS)) in a general older population.

Methods: 1,099 participants from a population-based older adult cohort study were recruited at baseline. 875, 768 and 563 participants attended years 2.6, 5.1 and 10.7 follow-up, respectively. Data were collected on demographic, psychological, lifestyle and comorbidities, blood pressure, glucose, triglycerides, and high-density lipoprotein (HDL) cholesterol. MetS was defined based on the National Cholesterol Education Program-Adult Treatment Panel III criteria. Radiographic knee osteoarthritis (ROA) was assessed by X-ray. Knee pain was measured by Western Ontario and McMaster Universities Osteoarthritis Index pain questionnaire at each time-point. Presence/absence of pain at the neck, back, hands, shoulders, hips, knees and feet was collected by questionnaire at each time-point. year study. Our findings support the role of SCTX-I and UCTX-II as Menzies Institute for Medical Research, Hobart, Australia; ${ }^{2}$ Monash University whether MetS and its components are associated with localised pain and

Group-based trajectory modelling was applied to identify pain trajectories. Multi-nominal logistic regression was used for the analyses.

Results: Of 985 participants included in this study, 32\% of participants had MetS and $60 \%$ had ROA at baseline. Three localised knee pain severity trajectories were identified: 'Minimal pain' $(52 \%)$, 'Mild pain' $(33 \%)$ and 'Moderate pain' (15\%). Three NPS trajectories were identified: 'Low NPS' (12\%), 'Medium NPS' (38\%), and 'High NPS' (49\%). In multivariable analysis without adjusting for central obesity, central obesity increased risk of belonging to both 'Mild pain' and 'Moderate pain' trajectories as compared to the 'Minimal pain' trajectory group, but MetS, hypertriglyceridemia and low HDL were only associated with 'Moderate pain' trajectory [relative risk $(R R): 1.67-2.26$, all $P<0.05$ ]. Similarly, central obesity was also associated with both 'Medium NPS' (RR 2.35, 95\% Cl 1.40-3.92) and 'High NPS' trajectories (RR $3.07,95 \% \mathrm{Cl} 1.85-5.08$ ) compared to 'Low NPS' trajectory group, whereas MetS was only associated with 'High NPS' trajectory (RR 2.60, 95\% Cl 1.54-4.41). These associations became weak and non-significant after further adjustment for central obesity.

Conclusion: MetS is predominantly associated with trajectories of localised and generalised pain through central obesity, suggesting that weight management is important in the prevention and therapy of pain over time.

Disclosure of Interests: None declared

DOI: 10.1136/annrheumdis-2019-eular.1245

\section{THU0421 EFFECT OF GAME BASED EXERCISE PROGRAMS ON PAIN, FUNCTIONAL MOBILITY AND BALANCE IN PATIENTS WITH KNEE OSTEOARTHRITIS: RANDOMIZED CONTROLLED STUDY}

DEMIRiz SERAP YILMAZ1', Ali Erdem Baki'. ' Zonguldak Atatürk Public Hospital, Physical Medicine and Rehabilitation, Zonguldak, Turkey; ${ }^{2}$ Zonguldak Bulent Ecevit University Faculty of Medicine, Physical Medicine and Rehabilitation, Zonguldak, Turkey

Background: Osteoarthritis is a chronic joint disease affecting the knee, hip and hand joints. Exercise is an integral component of conservative treatment for osteoarthritis. However, virtual reality applications using interactive games for rehabilitation have become a focus of interest in recent years.

Objectives: The aim of this study was to evaluate the effects of virtual reality games on knee pain, functional mobility and balance in patients with knee osteoarthritis.

Methods: Fifty patients who were complaining of knee pain, aged 40-70 years, and were diagnosed with Kellgren-Lawrence stage 2, 3, 4 idiopathic knee osteoarthritis was included. Patients were randomly assigned to two equal groups $(n=25)$ as the control and the study group. Age sex, weight, height, duration of illness was recorded for all patients. In the bilateral two-way knee radiography; Kellgren-Lawrence score of the knee with the higher visual analog scale (VAS) pain score was recorded. Conventional physical therapy program, associated with knee osteoarthritis exercises, was applied routinely to all patients with knee osteoarthritis for three weeks. In the study group, all patients received virtual reality game-based exercise program by using a game console. In both groups, baseline and final values of VAS score, osteoarthritis index total score (WOMAC), and intra-community balance and mobility scale (CB\&M) score of each patient were recorded. Mann-Whitney $U$ and Independent Samples $T$ tests were used for statistical analysis, and $p$ value less than 0.05 was considered significant.

Results: In both groups, significant change in VAS, WOMAC osteoarthritis index, and CB\&M score was observed $(p<0.05)$. In the study group, VAS, WOMAC osteoarthritis index, and CB\&M scores were significantly different than control group $(p<0.05)$. The differences between baseline

Abstract THU0429 - Table 1. Results of four separate multiple linear regression models with the WOMAC as the dependent variable. Regression coefficients with 95\% confidence intervals are shown.

\begin{tabular}{|c|c|c|c|c|c|c|}
\hline & \multirow[b]{2}{*}{ Parameter } & \multirow[b]{2}{*}{ Reference } & \multicolumn{4}{|c|}{ WOMAC } \\
\hline & & & Polyarthrosis & Hip OA & Knee and Hip OA & Knee OA \\
\hline \multirow[t]{2}{*}{ Model 1} & Age & per 10 years & $0,7(-0,6 ; 2,0)$ & $0,7(-1,2 ; 2,6)$ & $2,0(-0,5 ; 4,4)$ & $-0,1(-3,5 ; 3,3)$ \\
\hline & Gender: Male & Female & $-3,8(-6,7 ;-1,0)$ & $0,8(-3,5 ; 5,0)$ & $-3,7(-8,1 ; 0,6)$ & $-0,5(-7,6 ; 6,6)$ \\
\hline Model $2^{*}$ & $\mathrm{BMl}, \mathrm{kg} / \mathrm{m}^{2}$ & per 5 units & $4,4(3,2 ; 5,5)$ & $3,1(1,1 ; 5,2)$ & $4,2(1,8 ; 6,6)$ & $4,1(0,6 ; 7,6)$ \\
\hline Model 3* & Symptom duration & per year & $0,3(0,2 ; 0,5)$ & $0,4(0,2 ; 0,6)$ & $0,2(-0,0 ; 0,4)$ & $0,4(0,0 ; 0,8)$ \\
\hline Model $4^{*}$ & WHO-5 (0-100) & per $10 \%$ worsening & $4.4(3.8 ; 5.0)$ & $3.2(2.2 ; 4.1)$ & $4.1(3.3 ; 5.1)$ & $3.9(2.1 ; 5.9)$ \\
\hline
\end{tabular}

*Model adjusted for age, gender. BMI: Body Mass Index, OA: Osteoarthritis, WOMAC: Western Ontario and McMaster Universities Osteoarthritis Score. 